\title{
I. The series system in the spectrum of gold
}

\section{W.M. Hicks F.R.S.}

To cite this article: W.M. Hicks F.R.S. (1919) I. The series system in the spectrum of gold, Philosophical Magazine Series 6, 38:223, 1-31, DOI: 10.1080/14786440708635926

To link to this article: http://dx.doi.org/10.1080/14786440708635926

曲 Published online: 08 Apr 2009.

6 Submit your article to this journal $\pi$

Џll Article views: 2

Q View related articles $₫$ 
TH E

LONDON, EDINBURGH, AND DUBLIN

PHILOSOPHICAI, MAGAZINE

\author{
AND \\ JOURNAL OF SCIENCE.
}

[SIXTH SERIE8.]

JULY 1919.

I. The Series System in the Spectrum of Gold. $B y$ W. M. HICKs, F.K.S.*

7 THE spectra of the copper group of metals are interesting 1. in that, whilst they offer clear evidence of being built upon the same plan, they seem defective in representatives of the ordinary series lines and in some apparent deviations from relations indicated by other groups. They all show a pair of strong doublets in the ultra violet, a curions set of inverted $\mathrm{D}$ lines in the red-proved to belong to the $\mathrm{D}$ (or F) system by their Zeeman patterns-linked to associated lines by analogous $e$ and $u$ links, and $\mathbf{S}(2)$ doublets in the immediate ultra red also proved to belong to the $\mathrm{S}$ system by their Zeeman patterns. In copper and silver only two or three other lines have been assigned to $S$ and $D$ series, whilst in gold none have been allocated. The present communication attempts to throw some light on the series relations in gold. Although it does not pretend to be a thorough discussion of the material at disposal, certain definite results are arrived at, and some striking illustrations are afforded of general laws obtained in a series of papers published in the 'Transactions of the Royal Society' $\dagger$.

* Communicated by the Author.

$\dagger$ "A Critical Study of Spectral Series," Trans. Roy. Soc. I., 210 A. p. 57 (1910).-II., 212 A. p. 33 (1913).-III., 213 A. p. 323 (1914).-IV., 217 A. p. 361 (1918); and V., read Jan. 23 (1919). References in the present paper are made in [ ], thus-[IV. p. - ]

Phil. Mag. S. 6. Vol. 38. No. 223. July.1919. 
The arc spectrum of gold has been touched upon by me in [III. p. 405] and the spark spectrum has been more fully discussed in its linkage relations in [IV.]. It does not afford data for the direct determination of series constants. The two strong lines usually taken to correspond to the $\mathbf{P}(1)$ doublet give the doublet separation to be looked for in the P, S, or D series. The mean from measures by Kayser and Runge, Exner and Haschek, and by Eder and Valenta gives $\nu=3815.58$. The most reliable is that obtained from the inverse $D$ set above referred to, viz., $\lambda \lambda=5064 \cdot 75,6278 \cdot 37$ R.A., giving $\nu=3815.54$. These two were the only sets giving the separation in K. R.'s original measures of the arc spectrum. Since then E. \& H. have observed a line at $\lambda 4811 \cdot 81$, which K. R. appear to have missed and which has a separation 3815.57 with $\lambda 4665 \cdot 22$. Also Lehmann ${ }^{*}$ has observed in the ultra red a line at $7509 \cdot 8$ giving with K. R.'s 5837 a separation of $3813 \pm 13$, and allocated by bim to $\mathrm{S}(2)$ -an allocation justified by their position relatively to the corresponding lines in $\mathrm{Cu}$ and $\mathrm{Ag}$ and by the Zeeman pattern for 5837 as determined by Hartmann. The Zeeman effects for certain lines have been observed by Michelson †, Purvis $\ddagger$, and Hartmann . They are given in the notation suggested by me $\|$ in the following list:-

$\begin{array}{rcccl} & \lambda . & n . & \text { Hartmann. } & \text { Purvis. } \\ \mathrm{D}_{22}^{\prime}(1) & 6278 \cdot 37 & 15923 \cdot 38 & 0 / 5 ? & \\ p_{1}(1)-p_{1}(2) & 5957 \cdot 24 & 16781 \cdot 73 & 0 / 7 \frac{1}{2} & \\ \mathrm{~S}_{2}(2) & 5837 \cdot 64 & 17125 \cdot 54 & 4 / 8 & \\ \mathrm{D}_{11}^{\prime}(1) & 5230 \cdot 47 & 19113 \cdot 51 & 0 / 6 \frac{1}{2} & \\ & 4811 \cdot 81 & 20776 \cdot 50 & 0 / 5 ? & \\ & 4792 \cdot 79 & 20858 \cdot 97 & 0 / 6 & 0 / 6 \\ & 4065 \cdot 22 & 24592 \cdot 07 & & 0 / 3 \frac{1}{2} \\ & 3898 \cdot 04 & 25646 \cdot 81 & & 0 / 6 \frac{1}{2} \\ & 3804 \cdot 22 & 26279 \cdot 28 & & 0 / 6 \frac{1}{2} \\ & 3586 \cdot 66 & 27873 \cdot 33 & & 0 / 5 \\ & 3122 \cdot 88 & 32012 \cdot 59 & & 0 / 5 \\ & 3029 \cdot 32 & 33001 \cdot 33 & & 3 / 6 \frac{1}{2} \\ & 2913 \cdot 63 & 34311 \cdot 67 & & 0 / 6 \\ & 2748 \cdot 30 & 36374 \cdot 87 & & 0 / 6 \frac{1}{2} \\ \mathrm{P}_{2}(1) & 2676 \cdot 05 & 37357 \cdot 62 & & 3+/ 6\end{array}$

* Ann. d. Phys. xxxix. p. 75 (1912).

+ Nat. lix. p. 440 (1899).

+ Proc. Camb. Phil. Soc. xiv. p. 217 (1907).

$\S$ Dissertation Halle (1907). Sc. Abstracts.

|f Phil. Mag. xxxi. p. 171 (1916). 
The inverse $D$ set referred to are in W. N. :-

$$
\begin{array}{lcccc}
\mathrm{D}_{12} & -19738.92 & 3815.52 & -15923 \cdot 38(0 / 5) & \mathrm{D}_{22} \\
& 625 \cdot 41 & &
\end{array}
$$

The Zeeman effect $(Z=5 / 10)$ for the $D_{12}$ was not observed, but the others definitely belong to a $D$ (or possibly $F$ ) type. This and the corresponding sets in $\mathrm{Cu}, \mathrm{Ag}$ are quite out of step with the normal D lines. The satellite separation 625 , however, reproduces itself in a very striking manner in the spark linkages to which attention has been drawn in [IV. p. 385].

In [III. p. 405] an indirect attack on the problem was made by using the value of the oun as calculated direct from the atomic weight, $\delta=361 \cdot 8(\mathrm{~W} / 100)^{2}=1406.9$ and taking the lines 17125,20858 as $\mathrm{D}_{11}(2), \mathrm{D}_{22}(2)$, as they were in step with the undoubted corresponding $\mathrm{D}$ lines in $\mathrm{Cu}$ and $\mathrm{Ag}$. Their separation is $3733 \cdot 43$, producing therefore a satellite separation of $82 \cdot 11$, with a weaker $\mathrm{D}_{12}$ line too faint to have been observed. The observed Zeeman pattern for 20858 is $0 / 6$, which is that of a triplet $D_{22}$ line, in place of $0 / 5$ required for a doublet $D_{22}$, whilst 17125 is undoubtedly a $S_{2}$ line, although the pattern $4 / 8$ as observed is not perfectly clear. Since then 1 have found that Rydberg in his original memoir had attached the same two lines also to $D_{11}$ and $D_{12}$. It would appear that the $\mathrm{D}_{11}(2)$ and $\mathrm{S}_{2}(2)$ are almost coincident with 17125 , as the supposition appears to be justified by the results.

The method adopted was to apply the laws (1) that the separation 3815 was due to a displacement by a multiple of the oun on the $S$ or D limit, and (2) that the satellite separation of 82 was due to a displacement by an oun multiple in the $\mathrm{D}$ sequent. With this went the consideration that the limit would fall into step with those for $\mathrm{Cu}$ and $\mathrm{Ag}$, and would therefore be of the order $30000 \pm 1000$. The test (1) applied to these gave a series of possible limits between these values with $\Delta$ varying from $76 \delta$ to $81 \delta$. To each of these test (2) was applied, with the result that only two of them were found to satisfy the condition, viz. :-

$$
\begin{array}{lcc}
30819 \quad \Delta & =76 \delta \quad \text { sat. sep. due to } 24 \delta_{1}=6 \delta . \\
29465 \quad \Delta & =81 \delta \quad \text { " } \quad, \quad 28 \delta_{1}=7 \delta .
\end{array}
$$

The fact that 29465 is in due step with $\mathrm{Cu}=31515$, and B 2 
$\mathrm{Ag}=30644$, and some other considerations led to the adoption of the latter number. It was completely justified by the later discussion in [IV.], the links calculated on the foregoing: data being found to be satisfied with great exactness.

$$
\text { Taking } \quad \nu=3815.56, \quad \mathrm{D}_{1}(\infty)=29465 \cdot 18+\xi \text {, }
$$

$\mathrm{D}_{2}(\infty)=33280 \cdot 74+\xi+d \nu$, the mantissæ are

$$
\begin{aligned}
& \mathrm{D}_{1}(\infty) \quad 929298-32 \cdot 739 \xi, \\
& \mathrm{D}_{2}(\infty) \quad 815338-27 \cdot 273(\xi+d v),
\end{aligned}
$$

giving

$$
\Delta=113960-5 \cdot 466 \xi+27 \cdot 27 d \nu \pm 1=81 \delta \text {, }
$$

whence

$$
\delta=1406 \cdot 920 \pm \cdot 012-\cdot 067 \xi+\cdot 336 d v,
$$

the \pm ambiguities being due to uncertainties in final digits. when using 7-fig. logarithms with 7-fig. numbers.

With 20858.97 for $\mathrm{D}_{22}, \mathrm{D}_{12}$ is $v$ less. i.e. $17043 \cdot 41$. The mantissa of this satellite is $\cdot 971410-119 \cdot 60(\xi+\nu)+26 \cdot 3 p$, and of $\mathrm{D}_{11}=17125.54$ is $981279-120.80 \xi+16.9 p$, where $d \lambda=\cdot 05 p$ in each case, $05 \mathrm{~A}$ being in each case K. R.'s estimate of their possible error. The difference of these is

$$
\begin{aligned}
9872 \pm 1-1 \cdot 20 \xi+16 \cdot 9 p_{1}-26 \cdot 3 & p_{2}+119 \cdot 6 d \nu \\
= & 7\{1406 \cdot 920+3 \cdot 365 \pm \\
-14- & \cdot 171 \xi+2 \cdot 41 p_{1} \\
& \left.-3 \cdot 76 p_{2}+17 \cdot 1 d v\right\}=7 \delta .
\end{aligned}
$$

The difference of the two values of $\delta$ is

$$
3 \cdot 365 \pm \cdot 15-\cdot 104 \xi+2 \cdot 41 p_{1}-3 \cdot 76 p_{2}+16 \cdot 74 d \nu, .
$$

in which $d v$ is not greater than $0 \cdot 1$. The two values of $\delta$ therefore agree within quite easy limits of error, and the two results give no means of obtaining closer approximation. It is from this point that the present discussion starts. For this portion recourse must be had to the other general laws.

Now two well-established laws are that in the normal case (1) the mantissa of the extreme satellite of the first line in a $\mathrm{D}$ series is a multiple of $\Delta$, whilst (2) the mantissæ of succeeding orders differ from one another by multiples of the oun. But in all the elements of this group the mantissa of $\mathrm{D}_{12}(2)$ is decisively not a multiple of $\Delta$. The only explanation that appears to offer is that $m=2$ is not the first line, but that a set depending on $m=1$ exists in the far ultra red The evidence for this will be considered below. For our immediate purpose it should be noticed that the two relations above necessitate that the mantissæ of $D$ lines must. 
be multiples of the oun at least. Usually the oun is so small -or the multiple so large-that no further help to a closer approximation is attainable. In the case of $\mathrm{Au}$, however, the oun is so large that the actual multiples could be uniquely determined if the limit were known within about unity $(\xi=1)$. But in the present case the value of $\xi$ with our present knowledge may be considerable, and there will necessarily be an ambiguity of a few units.

With the above values of $\Delta$ and $\delta$,

$$
8 \Delta+49 \frac{1}{2} \delta=981326 \pm 8-47 \cdot 044 \xi+234 \cdot 7 d \nu,
$$

also mant. of $d_{1}=981279-120.80 \xi+16.9 p_{1}$.

These are equal within possible errors as they stand, but $\xi$ may be so large as to upset the agreement. If the multiple of $\delta_{1}$ is $y$ larger, $351.7 y$ must be added, and the condition of equality becomes

$$
351 \cdot 7 y+47 \pm 8+73 \cdot 76 \xi-16 \cdot 9 p_{1}+234 \cdot 7 d \nu=0 .
$$

If condition $\mathrm{A} \times 14$ be deducted from $\mathrm{B}$ the term in $d \nu$ disappears and

$$
\begin{gathered}
351 \cdot 7 y+75 \cdot 21 \cdot \xi-50 \cdot 6 p_{1}+52 \cdot 6 p_{2} \pm 11=0, \\
\xi=-4 \cdot 67 y \pm 1 \cdot 5 \quad(p \text { having max, values } \pm 1) .
\end{gathered}
$$

Then $d \nu=\cdot 2 \pm \cdot 02-\cdot 030 y-\cdot 14 p_{1}+\cdot 23 p_{2}=\cdot 2-\cdot 030 y \pm \cdot 4$.

The condition that $d v<\cdot 1$ is thus easily satisfied.

The results so far arrived at may be stated as follows:-

$$
\left.\begin{array}{rlrl}
\nu & =3815 \cdot 56+d \nu & & d \nu<\cdot 1 \\
\Delta & =113960-5 \cdot 466 \xi+x & & x \text { probably }<10 \\
\delta & =1406 \cdot 91-\cdot 0674 \xi+\cdot 0123 x & & \\
\xi & = \pm 1 \cdot 5-4 \cdot 674 y & & y=0, \pm 1, \pm 2 .
\end{array}\right\}
$$

It may be noted that if $y$ is as large as $2, \xi$ is altered by 9 and $\delta$ by $\cdot 6$. This would make $\delta$ so different from that determined directly from the atomic weight as to render it improbable, though not impossible ( $W=197 \cdot 20 \pm \cdot 07)$. Certainly $y$ cannot be so large as 3 . The only possible limits would then seam to be

$$
29465 \cdot 18 \pm 1 \cdot 5, \quad 29469 \cdot 85 \pm 1 \cdot 5, \quad 29474 \cdot 52 \pm 1 \cdot 5,
$$

with corresponding smaller values. By the preceding considerations the choice of the limit has been considerably restricted.

To arrive at a closer determination, we may attempt to obtain a more complete allocation of the D series, and have 
recourse to the existence of summation series if they show thenselves. This type of series has been shown to exist in $[\mathrm{V}$.$] . If the wave-number of a given series of the hitherto$ recognized type is written as the difference of two sequents $\mathrm{A}-\phi(m)$, it is there shown that in many cases at least a summation type $\mathrm{A}+\phi(m)$ also exists. When this occurs the mean of two corresponding wave-numbers gives the exact value of the limit. Unfortunately, it is also shown that displacements sometimes appear concurrently, especially in the summation type which require some care in applying the condition. In this particular case a displacement of one oun in the limit produces a change in it of 10.76 , and in the sequent a change which depends on the order $m$. For $m=2$, the lines just considered, it produces 2.90 in $d_{1}(2)$ and 2.93 in $d_{2}(2)$.

With the given $D(2)$, the region in which $D(3)$ is to be expected is narrowed down. The lines chosen were

$$
\begin{gathered}
{[22496 \cdot 77]} \\
32 \cdot 55+\cdot 5
\end{gathered}
$$$$
\text { (4) } 22529 \cdot 32 \pm \cdot 25 \text {, }
$$

in which the $D_{12}$ line is, as before, too faint to be seen, and is interpolated as $26312-\nu$. Its possible error is the same as that of 26312 . The oun displacement on the sequent produces $1 \cdot 228$. Consequently $28 \delta_{1}$ produces $32 \cdot 13$, which not only reproduces the observed satellite separation but gives the same oun multiple as in $m=2$, and lends additional support to the allocations. With $D_{11}(2)=17125$ and $D_{11}(3)=22529$ with the limit 29465 the formula for $D_{11}$ was determined and the wave-numbers up to $m=10$ calculated and compared with the observed lines. The result is given on the left hand of Table $I$. in which under any order the lines in succession correspond to $\mathrm{D}_{12}, \mathrm{D}_{11}, \mathrm{D}_{22}$. A search was then made for the corresponding summation or $\mathbf{D}$ lines which was successful. The results are entered on the right hand. The middle column gives the means of the corresponding $\mathrm{D}$ and $\mathrm{D}^{*}$ lines, which in the absence of displacement effects should be the true limits and the same in each order. They form the raw material for a closer discussion which follows. In the table calculated values are given in [ ], deduced values in italics.

* Summation series are denoted by clarendon type. The author lias found it convenient in writing to use capitals reversed right and left, ns in a looking-glass. 
Series System in the Spectrum of Gold.

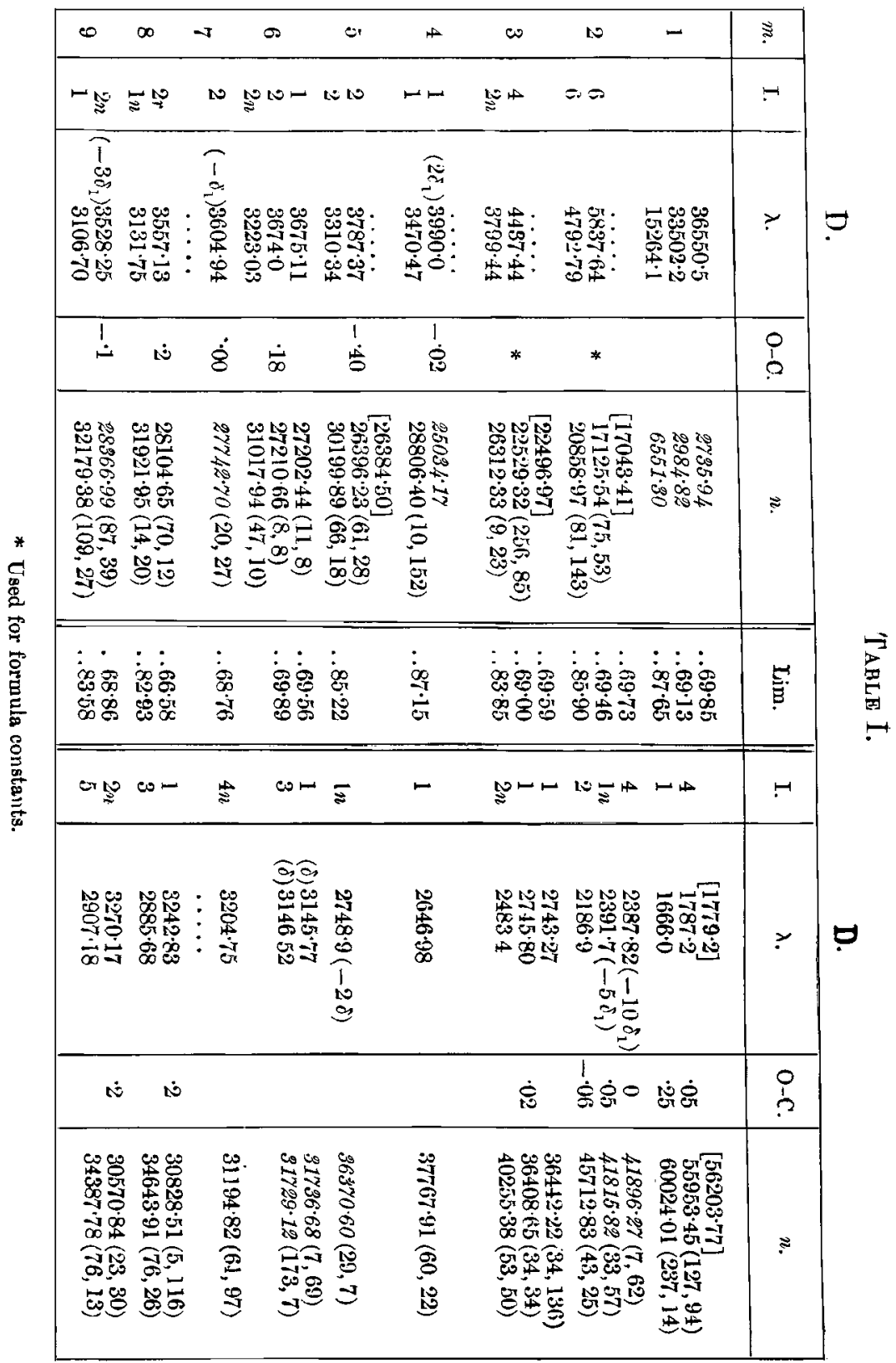


Let us take at first those data which depend directly on observed lines and do not require displacements for their interpretation. They are $\mathrm{D}_{22}(2)$ and the whole set for $m=3$. Their means point quite definitely to limits in the vicinity of 29469 and 33285 . In many portions of the Au spark spectrum the lines are excessively crowder, so that in those regions mere coincidences bave little value as evidence. Fortunately, however,, in these particular instances this is not the case. This is indicated by showing in brackets on the right of the lines the separation from the next observed lines on either side. In all the examples they are large, so that the combined evidence is convincing that the limits are close to the values given. It is striking, also, how closely they approximate to the second of the set of alternative values obtained above from quite independent considerations. It corresponds to putting $y=-1$ in the data under C, and makes them :

$$
\left.\begin{array}{rl}
\xi & = \pm 1 \cdot 5+4 \cdot 674 \\
\Delta & =113935+x \\
\delta & =1406 \cdot 60+0123 x \\
\mathrm{D}_{1}(\infty) & =26469 \cdot 85+\xi \quad \xi= \pm 1 \cdot 5 \\
\mathrm{D}_{2}(\infty) & =30285 \cdot 41+\xi .
\end{array}\right\}
$$

The formula for $D_{11}$, re-calculated with the new limit, is

$$
n=29469 \cdot 85-\mathrm{N} /\{m+\cdot 964130+\cdot 033168 / m\}^{2} \text {. }
$$

It gives for $m=4 \ldots 10$ wave-numbers of $25034 \cdot 05,26393 \cdot 42$, $27212 \cdot 05,27742 \cdot 76,28106 \cdot 24,28366 \cdot 01,28551 \cdot 06$. The question of the existence of lines corresponding to $m=1$ is of special importance for the reason mentioned above. Values extrapolated by the formula for $m=1$ rarely show any close agreement with observation, especially when, as now, they should occur in the far ultra red. In this case the law that the extreme satellite of the first line has its mantissa a multiple of $\Delta$ should give a very close approximate value for $D_{12}$ and $D_{22}$. This mantissa must be greater than that of $D(2)$, and it is natural to take for the first experiment the multiple just larger-in this case $9 \Delta$-and then seek for evidence as to its existence or not.

The value of $9 \Delta$ is $1 \cdot 025370+9 x$. This gives

$$
\begin{aligned}
& \mathrm{D}_{12}=2733 \cdot 70+\xi+\cdot 237 x, \\
& \mathrm{D}_{22}=6549 \cdot 06+\xi+\cdot 237 x .
\end{aligned}
$$


Paschen, who has measured lines in the far ultra red for $\mathrm{Cu}$ and $\mathrm{Ag}$, has unfortunately not dealt with $\mathrm{Au}$, so that direct comparison with observation is not possible. There are, however, indirect methods which may be applied, viz., the possible existence of summation lines (far in the violet), the method of sounding as exemplified in [IV., V.], and the presence of Ritz combination lines. In addition the $d(1)$ sequents should be the limits of a doublet $F$ series. The corresponding $\mathbf{D}$ lines should be at

$$
\begin{aligned}
& \mathrm{D}_{12}=56206 \cdot 00+\xi-\cdot 237 x, \\
& \mathbf{D}_{22}=60021 \cdot 56,
\end{aligned}
$$

of which the $D_{12}$ should be considerably weaker than $D_{22}$, and a strong line intermediate to these should be expected as a representative of the $D_{11}$ line. The separation of the $D_{12}$ and $D_{11}$ gives the doublet separation of the $F$ series and should show a high occurrency. Fortunately we possess measures so far down in the ultra violet by Handke *, and amongst them is $60024 \cdot 01.3 \cdot 6$ of intensity 1 , but no 56206 . There are several strong lines in a suitable position for $D_{11}$, but 55953 gives a satellite separation of 250. This separation occurs so frequently throughout the whole spectrum as to fit in well with the condition for a $D_{11}$ line. The whole set would then be arranged as follows :-

$\begin{array}{llc}2733 \cdot 70+\xi+\cdot 237 x & 29471 \cdot 07+\frac{1}{2} \xi+11 x \pm 1 \cdot 8 & {[56208 \cdot 45 \pm 3 \cdot 6]} \\ \mathbf{2 5 2 \cdot 5 5} & & \mathbf{2 5 5 \cdot 0 0} \\ {[2986 \cdot 25+2 \xi \mp 3 \cdot 1]} & 29469 \cdot 85+\xi & (4) 55953 \cdot 45 \pm 3 \cdot 1 \\ 6549 \cdot 06+\xi+\cdot 237 & 33286 \cdot 53+\frac{1}{2} \xi \pm 1 \cdot 8 & \text { (1) } 60024 \cdot 01 \pm 3 \cdot 6\end{array}$

A displacement of $28 \delta_{1}$ or $\tau \delta$ in the sequent produces a satellite separation of $258 \cdot 03$, or $27 \delta_{1}$ of $248 \cdot 88$. The possible errors in the observed do not enable us to settle "hich is the more probable. We shall see later, when dealing with the $\mathrm{F}$ series, that these separations are reproduced. The appearance of the summation lines in the exact positions to be expected, their relative intensities, and with a satellite separation depending on the same oun-multiple displacement as for orders $m=2,3$, together form weighty evidence in favour of the existence of the $D(1)$ lines as calculated.

But we can obtain further evidence by sounding for the actual $\mathrm{D}$ lines themselves. This methed has been developed in [V.] and depends on the linkage laws developed in [IV.]. The $e, u, v$ links in Au are so large that they can stretch from

* Dissert. Berlin. The measures used are quoted from Lyman. 
either of the above lines into the observed regions. Their values $e=p_{2}(1)(-2 \Delta)-p_{2}(1)(2 \Delta), \quad u=s(1)-s(1)(\Delta)$, $v=s(1)(-\Delta)-s(1)$ can be calculated from the values of $\Delta$ and $p(1)$ given above and with the $S(\infty)=29469 \cdot 85+\xi$ are $e=17252 \cdot 31, u=11340 \cdot 77, v=14534 \cdot 05$, with respective changes $10 \cdot 25,9 \cdot 17,13 \cdot 32$ per oun-modification. It is shown in [IV.] that each series line is linked to long sets of other lines by these separations, and that successive $e, u$ or $e, v$ links are very common. If, then, to an unseen line $n$ we observe another at say $e+n$, it is an indication-though not a proofthat the line $n$ exists. If several such links are found together the evidence is greatly strengthened. When a link is added to a wave-number $n$ it is written after it, if subtracted then it is written before it. Thus $2733 . e=19987$, or $e .19987=2733$. In the following the calculated values are given in [] and the observed set beneath (with separations. from next observed lines as in Table I.).-

$v, 2733 \cdot 70 . v=[17667 \cdot 75]$.

(4) $17675 \cdot 53(23,16) \quad 3814 \cdot 84 \quad(21490 \cdot 37)=(3 r) 21499 \cdot 65\left(-\delta_{1}\right)(66,41)$

$252 \cdot 23$

(1n) $17927 \cdot 76(7,31)$

$u, 2733 \cdot 70 . u=[14074 \cdot 47] ; 6549 . u=[17889 \cdot 77]$.

outside obs. $\quad(4 \mathrm{r}) 17890 \cdot 37(11,7)$

$e, 27337 . e=[19986 \cdot 0]$.

$\begin{array}{lll}(2) 19988.97(14,112) & 3816.59 & (2 n) 23805 \cdot 56(45,75)\end{array}$

[20240] not obs.

The $v$ sounding gives a satisfactory complete set if the $\mathrm{D}_{22}$ be really an oun displacement on the $D_{2}$ sequent as indicated. The oun on $d_{2}$ produces a change of $9 \cdot 285$ and on $d_{1}$ of $9 \cdot 155$. As additional evidence for the $D_{22}(1)$, may be added the $e+v$ sounder, i.e., the $e$ sounder for the above set. We find $e(1) 38746^{\circ} 77 \pm 1 \cdot 5=21494 \cdot 46 \pm 1 \cdot 5$. The next line to this is (1) $38757 \cdot 29 \pm 1 \cdot 5$, which is equivalent to an oun displacement on the sequent, and is the corresponding line linked by $e$ to $21504 \cdot 98$.

The $u$ link is not large enough to reach the $\mathrm{D}_{12}, \mathrm{D}_{11}$ lines. It indicates, however, as is seen, $\mathrm{D}_{22}$. This line 17890 is also associated with two lines ahead by $e$ and $v$ links.

In the $e$ sounding again we get a good indication. There is no observed line, however, corresponding to $\mathrm{D}_{11}(1) \cdot e$. The nearest is $(2 n) 20200 \cdot 35(100,117)$. Numerically it is 
$\left(2 \delta_{1}\right) \mathrm{D}_{11}(1)\left(2 \delta_{1}\right) . e$, giving $\mathrm{D}_{11}(1) . e=20240^{\circ} 18$. Although exact, the double displacement is of small evidential value unless supported by independent considerations.

In connexion with the third kind of tests I have noted the two combinations $d_{1}^{\prime}(1)-\bar{d}_{2}(1), \quad d_{1}^{\prime}(1)-d_{1}(1)$, where $d^{\prime}$ denotes the sequent for the inverse $D$ set already referred to. Since $-D^{\prime}{ }_{11}(1)=p(1)-d^{\prime}{ }_{1}(1)$ and $D_{11}(1)=p(1)-d_{1}(1)$, it follows that $d^{\prime},(1)-d_{2}(1)=\mathrm{D}_{11}^{\prime}(1)+\mathrm{D}_{12}(1)=19113.53+$ $2733 \cdot 7=21847 \cdot 2$; this is observed at $(2) 21846 \cdot 41$. So $d_{1}^{\prime}(1)-d_{1}(1)=\mathrm{D}_{11}^{\prime}(1)+\mathrm{D}_{11}(1)=19113 \cdot 53+2986=22099$, which is observed at $(4 n) 22102 \cdot 18$ within possible limits. With the first it would give the satellite separation as $255 \cdot 77$.

The cumulative evidence of these three independent considerations is convincing that the $D(1)$ lines as suggested really exist, and that the rule for the extreme satellite mantissa is not broken in the case of $\mathrm{Au}$, as it apparently was when the old observed $\mathrm{D}(2)$ line was regarded as belonging to the first set. The consideration of the $F$ series which follows will give direct proof that the $d(1)$ sequence at least exists, but before taking up that question it is necessary to complete the discussion of the results of Table $I$. which raise points of importance not only in connexion with the properties of D series, but of those of displacement in general.

$m=2$. No direct lines are found for the weaker $D_{12}$ and $D_{12}$, nor for $D_{11}$. Rydberg had allotted (4) 17050.97 to $D_{12}$, but $K$. R. have pointed out that its separation from $\mathrm{D}_{22}=20858$ is too large. The true value cannot deviate more than 32 from $17043 \cdot 41$. This differs by $7 \cdot 56 \pm \cdot 46$ from 17050 . The oun displacement on $d(2)$ produces 2.93 , so that 7.56 is as far as it can be from a value produced by $2 \delta_{1}$ or $3 \delta_{1}$ and much beyond the possible errors of $\pm \cdot 46$. The line in question therefore cannot be a simple displacement of $\mathrm{D}_{12}(2)$. Since the oun on the limit produces $10 \cdot 76$, it may be $\left(\delta_{1}\right) \mathrm{D}_{12}(2)\left(\delta_{1}\right)$, which is separated from $\mathrm{D}_{12}$ by $10 \cdot 76-2 \cdot 93=7 \cdot 83$, but is probably unrelated to the $\mathrm{D}$ series. The $D_{11}$ and satellite appear each displaced into at least two. others. Thus

$$
\begin{array}{rrrrr}
(4) 41866 \cdot 75+87 p & \text { as } & \mathbf{D}\left(10 \delta_{1}\right) & \text { gives } & \mathbf{D}_{12}=41896 \cdot 05+87 p \\
(2) 41928 \cdot 72+87 p & , & \mathbf{D}\left(-11 \delta_{1}\right) & \Rightarrow & \mathbf{D}_{12}=48896 \cdot 49+87 p \\
(1 n) 41798 \cdot 68+1 \cdot 7 p & \prime & \mathbf{D}\left(5 \delta_{1}\right) & \Rightarrow & \mathbf{D}_{11}=41813 \cdot 18+1 \cdot 7 p \\
(5) 41859 \cdot 04+1 \cdot 7 & \Rightarrow & \mathbf{D}\left(-16 \delta_{1}\right) & \Rightarrow & \mathbf{D}_{11}=41812 \cdot 64+1 \cdot 7 p
\end{array}
$$

For $m=3$ the satellite separation is due to $26 \delta_{1}$ in line 
with those for $m=1,2$. All the set appear except the weak line $D_{12}$, and it is at the same time instructive to notice that there appear no signs of displaced lines. For $m=4$, on the contrary, there are possible representatives only of $D_{22}$ and $D_{22}$, but there are clear evidences of extensive displacements. The calculated value for $\mathrm{D}_{11}$ is $25034 \cdot 05$. This has a $u$ link to (4) $36374 \cdot 87$, for which the Zeeman pattern has been observed by Purvis. It is represented by $0 / 6 \frac{1}{2}$, the proper pattern for a $D_{11}$ line. Displacement certainly alters the Zeeman pattern-witness, as an instance, the different forms for satellite lines. If this indication that linkage leaves the Z-patterns unaffected is sustained by further evidence, it would be of fundamental importance for spectral theory. The normal line 20534 itself appears split up into a number of others all showin $r$ displacements by an oun multiple in the limit. We find in succession the lines in the following list, in which are given the suggested displacements, and the $\mathrm{O}-\mathrm{C}$ values on the supposition that the true $\mathrm{D}_{11}(4)$ should be $25034 \cdot 24$ :-

$$
\begin{array}{rcc}
\text { (1) } 25012 \cdot 07 & \left(2 \delta_{1}\right) \mathrm{D}_{11} & \cdot 11 \\
{[25034 \cdot 05]} & {\left[\mathrm{D}_{11}\right]} & \cdot 03 \\
\text { (1) } 25045 \cdot 39 & \left(-\delta_{1}\right) \mathrm{D}_{11} & -\cdot 06 \\
\text { (1) } 25055 \cdot 69 & \left(-2 \delta_{1}\right) \mathrm{D}_{11} & \cdot 01 \\
\text { (1n) } 25077 \cdot 80 & \left(-4 \delta_{1}\right) \mathrm{D}_{11} & -\cdot 08
\end{array}
$$

The two lines suggested in the Table for $D_{22}, D_{22}$ are probably not the normal, but may be displaced. They would give a satellite separation of $43 \cdot 40$, much too large to be in step with those for $m=1,2,3$. Here $28 \delta_{1}$ would produce a separation of $17 \cdot 5$. The general disintegration of the $D(4)$ set would seem to be indicated by the existence of fragments of other sets in the neighbourhood. For instance, we find
(3n) $2.5170 \cdot 53$
$2947 \% \cdot 88$
(1r) $33775 \cdot 23$
3813.7\%
$3814: 52$
(1) $28984 \cdot 23$
33286.99
(1) $37589 \cdot 75$

Here 25170 has the normal limit.

In $m=5, \mathbf{D}_{22}$ is represented by two displaced lines, viz.,

$$
\begin{aligned}
\text { (5) } 36374 \cdot 88 \text { as } \mathrm{D}_{22}(-3 \delta) & \text { gives } \ldots 70 \cdot 52 \text {, } \\
\text { (1n) } 36367 \cdot 70 \text { as } \mathrm{D}_{22}(2 \delta) & \text { gives } \ldots 70 \cdot 60 \text {, }
\end{aligned}
$$

but the first is $D_{11}(4)-u$, and is too strony to represent $D_{22}$. It forms a merely numerical coincidence. 
In $m=6$, the displaced $\mathbf{D}_{12}, \mathbf{D}_{22}$ would seem justitied by their order of intensities and same displacement $=\delta$.

In $m=7,3604 \cdot 96$ is exactly the calculated $\lambda$ for $\left(\delta_{1}\right) D_{1}(7)$. But this line is also, as will be seen later, the combination $p_{1}(1)-p_{1}(7)$. The values coincide. Whether the observed is either or both there is nothing to decide.

$m=8$. The calculated $D_{11}$ gives a better $\nu$ with $D_{22}$, and a closer value to the mean limit.

The foregoing discussion supports conclusively :

(1) The value of the limit $\mathrm{S}(\infty)=\mathrm{D}(\infty)$ is 29469.85 \pm 1.5 .

(2) The existence of summation $D$ series.

(3) The existence of extreme ultra red D lines, depending very closely on $9 \Delta$ as the mantissa of the $d_{2}$ sequent.

(4) A satellite separation about 249 or 258 due to displacements of $27 \delta_{1}$ and $28 \delta_{1}$.

With this limit and its uncertainty $1 \cdot 5$, and $v=3815 \cdot 56+d v$, $d \boldsymbol{v}<\cdot 1$,

$$
\begin{aligned}
\Delta & =113935+27 d \nu-5 \cdot 46 \xi=113935+x, x<10, \\
\delta & =1406 \cdot 604+\cdot 123 x \\
f(8 \Delta) & =30017 \cdot 06-\cdot 251 x, \\
f(9 \Delta) & =26734 \cdot 86-\cdot 237 x .
\end{aligned}
$$

It should be remembered, however, that the value of the oun as determined from the $S(\infty)$ and the doublet or triplet separation appears often to show an indication of being very slightly smaller than when determined from $D$ or $F$ data, although the latter are very consistent amongst themselves. It is possibly due, if it is real, to a difference in the value of $N$ between $P$ and $D$ sequences. In any case it is always extremely small. Here the value from $D$ and $F$ data would depend on the $D(1)$ lines, and we have no direct observations to give their values with sufficient exactness to test. So far as evidence is at disposal it must come from the sounding with $e, u, v$ links given above, and these point to a slightly higher value of the $D$ lines than those calculated. In fact, the following arrangement would fit all the sounding indications, within error limits. The observed lines are supposed to be corrected by amounts less than the possible 
errors and are shown as alterations on the observed measures :-

D.

$3815 \cdot 56\left\{\begin{array}{c}2735 \cdot 94 \\ 248 \cdot 88 \\ 2984 \cdot 82 \\ 6551 \cdot 30\end{array}\right.$

D.

[56203777]

$248 \cdot 88$

$29469 \cdot 85$

39285:41

$$
\begin{array}{ll}
55953 \cdot 45+1.44 & (p=\cdot 5) \\
60021 \cdot 04-4.68 & (p=1 \cdot 3)
\end{array}
$$

This would give $d_{2}(1)=29469 \cdot 85-2735 \cdot 94=26733 \cdot 91$. It may be taken as this with an uncertainty at present of \pm 4 . This makes $\Delta=111940, \delta=1406 \cdot 78$ or within the limits of the direct determination from $\mathrm{S}(\infty)$.

The $\mathrm{F}$ and $\mathbf{F}$ series. These series have as their limits the first $\mathrm{D}_{11}, \mathrm{D}_{12}$ sequents. With our new $\mathrm{D}(1)$ lines or the $d_{2}$ sequent with a mantissa $9 \Delta$,

$$
\mathrm{F}_{2}(\infty)=d_{2}(1)=26733 \cdot 9-\cdot 237 x \quad x \operatorname{not}>10 \text {. }
$$

The D satellite displacement of $27 \delta_{1}$ gives a separation of 248.89 , or $28 \delta_{1}$ of 258.05 . We should expect, therefore, doublet series with these separations on both sides of 26734 , appertaining to $F_{1}(\infty)=26486$ or $26476 \cdot 81$. We find on inspection a very large number indicating a profusion of displacements, and the lines in consequence so crowded that it is difficult to determine definitely whether numerical coincidences correspond to real relationships or not. For our present purposes we desire to obtain the $\mathrm{F}_{2}(1)$ lines. Now, like the $d$ sequences the $f(1)$ sequence also has its source in a mantissa which is a multiple of $\Delta$ and at the same time is a large fraction approximating to unity. Here, then, the multiple can only be 9 or 8 , or possibly 7 or 6 -or there may be, as in the rare gases, several groups depending on both. In other elements we find indications of $\mathrm{F}$ satellites depending only on small displacements of order $2 \delta_{1}$. Also the lower orders are often subject to very large $\Delta$ displacements. We proceed to discuss the two cases of $9 \Delta$ and $8 \Delta$ in order :-

Case I. $f(1)=\mathrm{N} /(9 \Delta)^{2}$. In this case $d_{2}(1)$ and $f(1)$ are identical and equal to $26733 \cdot 96 \pm 2$. The expected lines should therefore be :

$$
\begin{array}{ll}
\mathrm{F}_{2}(1)=0 & \mathbf{F}_{2}(1)=53467 \cdot 8 \pm 4, \\
\mathrm{~F}_{1}(1)=-248 \cdot 89 & \mathbf{F}_{1}(\mathbf{1})=53218 \cdot 9 \pm 4 .
\end{array}
$$

$\mathrm{F}_{2}(1)=0$ would correspond to no oscillation and probably to instability, and lead to an expectation of displacement. 
For direct evidence we must look, as before, for $\mathbf{F}$ lines in the ultra violet. There are no lines at the places indicated. The nearest are (6) $53401 \cdot 69+2 \cdot 9 p,(-70,183)$, and (2) $53151 \cdot 91+2 \cdot 9(-174,32)$ with the proper separation of $249 \cdot 78 \pm$. If they are the sought $F$ lines the complete set would be :

$$
\begin{array}{rrrr}
\mathrm{F}_{1} & -181 \cdot 9+x & 26485 \cdot 0 & 53151 \cdot 91 \pm 2 \cdot 9, \\
\mathrm{~F}_{2} & 66+x & 26733 \cdot 9 & 53401 \cdot 69 \pm 2 \cdot 9,
\end{array}
$$

with $x$ of order \pm 4 , depending on obs. errors $2 \cdot 9 p$ and limit uncertainty. These are $66 \pm$ less than the calculated values and can be explained by a $+7 \delta_{1}$ displacement in the sequent, which produces a separation of 64.75 . The $u, v$ links are too small to reach into the observed region. We have no knowledge as yet as to how links should be attached to wavenumbers which formulæ give as negative, but we should expect doublet separations to be conserved. We should then look for lines, with added and with subtracted links as follows :-

$$
\begin{aligned}
& \mathrm{F}_{1} \quad e-181 \cdot 9+x=17071 \cdot 8+x, \quad-e-181 \cdot 9=17435 \cdot 7+x, \\
& \mathrm{~F}_{2} e+66+x^{\prime}=17319 \cdot 8+x^{\prime},-e+66=17187 \cdot 8+x^{\prime} .
\end{aligned}
$$

Now we find in this neighbourhood a large number of lines with all the signs of being débris, so to say, of a shattered system, viz., weak lines of the same nature and separated by multiples of the same numbers. Taking the lines as they occur in order they may be arranged as follows:-

$$
\text { e. } \mathrm{F}_{2} \text { e. } \mathrm{F}_{1} \text {. }
$$

(2n) 1741002

(1n) 1717850

$9 \cdot 3$

$\left[17187 \cdot 8+x^{\prime}\right]$

$29.57=6 \times 4.93$ $[174357+x]$

$251 \cdot 8-x^{\prime}(2 n) 17499 \cdot 59$

499

(1n) $17444 \cdot 58$

1148

$251 \cdot 51$

$251 \cdot 44$

(3) $17456.06 \quad 251.04$ (11i) 17606.02

$4 \cdot 19$

(1r) $17700 \cdot 21$

6.89

(4) 17675.53 are. [17684:6] (1n) $17691 \cdot 10$

\subsection{2}

(3r) $17707 \cdot 10$

4.39

(3n) $17711 \cdot 49$

$9 \cdot 11$

(3) 1772069 
$\mathrm{F}_{1}$.e.

(4) $17050 \cdot 97$ arc.

20.8

$[17071 \cdot 8+x]$
$\mathrm{F}_{2}$.e.

(2n) $17269^{\circ} 10=e+15$

\section{$64: 84$}

$\left[17319 \cdot 8+x^{\prime}\right] \quad\left\{\begin{array}{rr}242 \cdot 4 & \text { (1u) } 17562 \cdot 23 \\ 254 \cdot 1 & \text { (3) } 17573 \cdot 91\end{array} 11 \cdot 68\right.$

$262.1 \quad(1 n) 17333.94$

15.79

(1n) $173+9 \cdot 73$

6.23

(5) $17355 \cdot 96$

$251.2 \quad(1 n) 17601.92$

It will be noticed, the same weak and nebulous character of the lines, the frequency of separations depending on multiples of $4 \cdot 9$, and also similar analogies in the two sets, e.g. the changes from a sharp line to a nebulous one with sep. $11 \cdot 6$ or in that neighbourhood at 17456,17573 . In relation to the whole set it sbould be remembered that we should expect the $27 \delta_{1}$ or $28 \delta_{1}$ displacements of the D series to be repeated on the limit, or on the $f$ sequent. In this connexion the following data may be given :-

$$
\begin{array}{cccc}
+27 \delta_{1} & -27 \delta_{1} & +28 \delta_{1} & -28 \delta_{1} \\
248.91 & 252 \cdot 39 & 258.05 & 261.80
\end{array}
$$

The effects are capable of so many interpretations that it is impossible to draw any certain conclusions. A few remarks, however, may not be out of place :-

(1) $f(1)$ is taken as $d_{2}(1)\left(7 \delta_{1}\right)$, so that the set are $d_{1}(1) \pm f(1)$ and $d_{2}(1) \pm f(1)$.

(2) 17178 is either $e \cdot\left(\delta_{1}\right) F_{2}$ or $e \cdot F_{2}\left(-\delta_{1}\right)$.

(3) In 17269 the original displacement of 64.8 is reproduced.

(4) It is difficult to explain the differences 4.9 by oun displacements. They could possibly be explained by the oun if these series depended on a Rydberg constant $4 \mathrm{~N}$, as in the enhanced series of the alkaline earths, and there are some independent indications pointing to this.

(5) The important consequence to be drawn is that they afford evidence of the extreme disruption of a set, and of the nature of the complicated displacement systems called into play and which require disentanglement.

We might now attempt to allocate sets of lines to stand in the relation of higher orders to the series. They exist, but 
I omit reference to them, as for the present purpose-that of illustration of method and the more exact determination of the gold oun-the next set in which $f(1)$ depends on $\mathrm{N} /(8 \Delta)^{2}$ is preferable and is sufficient.

Case II. $f(1)=\mathrm{N} /(8 \Delta)^{2}$. Here $f(1)=30017 \cdot 06-\cdot 251 x$ with the oun displacement producing a change of $11 \cdot 046$. With the $d(1)$ value $F(\infty)$, viz., $26734 \cdot 86-\cdot 237 x$, the calculated values of the set for $m=1$ are

$$
\begin{array}{llll}
\mathrm{F}_{1} & -3531 \cdot 20 & 56502 \cdot 92 & F_{1}, \\
\mathrm{~F}_{2} & -3282 \cdot 20 & 56751 \cdot 92-.488 x & F_{2}
\end{array}
$$

For the $\mathbf{F}$ lines only (1) $56744 \cdot 03+3 \cdot 22 p$ is observed. It should be normally a strong line. There is a strong line at (5) $56721 \cdot 50$ separated by $22 \cdot 53$, and as $2 \delta_{1}$ in the sequent produces 22.09 it is probably $\mathbf{F}_{2}(1)\left(2 \delta_{1}\right)$. The $\mathbf{F}_{1}$ should be 56495 (not seen). But there is again a strong line (5) 5656109,66 ahead. This may be either $6 \delta_{1}(66 \cdot 27)$ on the sequent or the $7 \delta_{1}\left(64^{\circ} 0\right)$ on the limit (similar to Case I.). The only interest in these at present is that if displaced $F$ lines, tho weakness of the normal ones is explained.

For evidence of the $\mathrm{F}$ lines we must again have recourse to sounding. The $u$ links are too small to reach. The $v$ links land close to a doublet with the proper separation, viz. :

$$
\begin{array}{lll}
\text { (1n) } 18217 \cdot 03+\cdot 15 p & 250.53 & \text { (3) } 18467 \cdot 56+\cdot 15 p
\end{array}
$$

with again several lines intensity $(1 n)$ near 18217 , suggesting other displaced lines with consequent weakness. Subtracting $v$ gives as the F lines $3283,3533 \cdot 51$. Treating the series as $\mathrm{N} /(\mathbf{9} \Delta)-\mathrm{N}(8 \Delta)$ gives-with the observed $\mathbf{F}_{2}-$

$$
\begin{array}{rcc}
-3533 \cdot 51 & {[j 6493 \cdot 52} \\
250 \cdot 51 & 250 \cdot 51 \\
-3283 \cdot 00 & 26730 \cdot 52+1 \cdot 6 p & 56744 \cdot 03+3 \cdot 22 p .
\end{array}
$$

In the absence of observation in the ultra red this set cannot be considered as conclusive, although it satisfies the conditions. If it be taken as real, the observed $56744 \cdot 03$ is the value calculated above and should give the value of $x$. That is

$$
\begin{gathered}
56744 \cdot 03+3 \cdot 22 p=56751 \cdot 92-\cdot 488 x, \\
\text { whence } \quad x=16 \cdot 16-6 \cdot 60 p .
\end{gathered}
$$

This gives a closer value to $\Delta$ as depending on the $\mathrm{D}, \mathrm{F}$ data. If it is the same as from $\mathrm{S}(\infty)$ and $\nu, x<10$ or $p$ should be about 1 -or the observed measure of the W, L should be -1 A less. The possible error is certainly greater than this. Provisionally we will take $p=1, x=9 \cdot 5+x$, where now $x=0$

Phil. Mag. S. 6. Vol. 38. No. 223. July 1919. 
if the ouns calculated from $S$ and D are the same, but may he anything between \pm 6 if not.

This makes

$$
\Delta=113951-6 \cdot 60 p,
$$

from which

$$
\mathrm{N} /(9 \Delta)^{2}=26731 \cdot 03+1.56 p .
$$

It will be noted that allowance being made for uncertainty in $v$ this is the mean limit given by the observations. It points to the fact that the link is not modified and the observed line 18217 has suffered no displacement in the linking. If so, any differences occurring can only arise through observation errors. Take the natural supposition that the two determinations of the oun are the same. Then, as we have seen, $p$ is about 1 , say $\cdot 9$, so that $x=10$. The link $v$ is increased by 98 or say 1 . The possible error in 18217 is $\cdot 15 p^{\prime}$. Hence $\mathrm{F}_{2}=-3282 \cdot 00-\cdot 15 p^{\prime}$ and the mean limit is now $26732 \cdot 66-.07 p^{\prime}$, as ayainst the value directly calculated from $\mathrm{N} /(9 \Delta)^{2}$ of $26732 \cdot 43$, wr exact equality.

This is not a mere meticulous refinement. The analysis is exact when the data are definite. The difficulty in general is that on account of the numerous displacements in two sequents, modified links, and other changes, the data themselves are subject to indefiniteness.

Before attempting to allot lines for higher orders it may be advisable to consider what we are to expect. The D and $\mathrm{F}$ systems of lines have much in common-indeed, it may ultimately be found on more complete knowledge that the $f-$ are only another set of $d$-sequences. There is considerable evidence ${ }^{*}$ that the $d$-sequences are not representable by a continuous mathematical expression, but that they may approximate to values so represented. In the $\mathrm{F}$ system, wherever they have been discussed in detail, this tendency is still more marked. There is indisputable evidence that what we should regard as a normal hine is often accompanied by a number of others displaced in definite ways, that its intensity is then diminished, and, indeed, that it is itself, in that case, frequently absent. In some instances the whole set for a given order $(m)$ may be absent and represented either by another strong set related to it by a displacement of several multiples of $\Delta \dagger$, or by a congery of membra disjecla displacod by various oun multiples. One is even tempted to suspect that the possible lines of a spectrum-at least in the $\mathrm{D}$ and $\mathrm{F}$ systems-are built $\mathrm{up}$ by the addition to the mantissa of successive multiples of the oun, and that the

* See, for instance, Astro. J. xliv. p. 229 (1916).

$\dagger$ E. g. alkaline earths [III. p. 383 seq.], the rare gases [V.]. 
intensities-or the chance of the particular configurations corresponding to those multiples existing-alter with each addition, rising to maxima under certain as ret unknown conditions. That sometimes such a displacement suddenly confers increased intensity is certainly true. This whole question is a most interesting one and of fundamental importance in any theory of spectral origins. Before it can be profitably discussed, however, a greatly increased collection of raw material bearing on this point must be obtained. The spectrum of gold seems especially promising for a more exhaustive investigation under this head.

In the following list many lines have been. included to illustrate the effects of displacement as above indicated. Several of the individual illustrations are doubtless spurious and mere numerical coincidences. This may be specially the case with lines occurring in the region embracing $W, N 25000$ to 28000 (say, $\lambda \lambda 4000$ to 3500 ), which is exceedingly crowded owing to this cause, containing as it does lines belonging to different sequents and different limits. It is, however, the number of such coincidences which give evidence as to the general existence of the displacement effect. Their consideration is, however, of importance from another aspect. A line associated by one of the usual links to an unseen one is evidence in support of the possible existence of the unseen, but it does not give the power in general of exactly determining its measure, owing to uncertain linkage modifications. On the other hand, an observed line satisfying a condition of displacement from a suspected one, gives no reliable evidence that such a suspected line can actually exist, but it does give evidence as to its theoretical measure, if it were capable of being emitted. This point is of importance in settling the mean limits as determined from the means of difference and summation lines, i.e. limit $=\frac{1}{2}(F+F)$, where one of the lines in question is determined by displacement from an observed set.

In the following table sets of lines have been allotted whose mantissæ in the different orders $(m)$ are of the same order of magnitude-a condition which normal series lines must conform to. Also a considerable number of other lines have been added where they seem to show the presence of displacement, especially where what should be a strong line appears represented by a number of weak ones of similar character. Under each order is inserted the change produced in the sequent by the displacement of one oun. The corresponding change on the limit is $9 \cdot 14$. Deduced values are in () . 
$\stackrel{\frac{1}{3}}{\frac{\infty}{2}}$

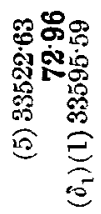

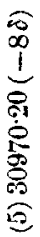

$\frac{5}{\infty}$
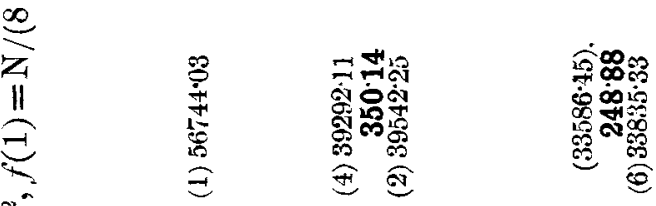

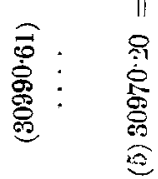
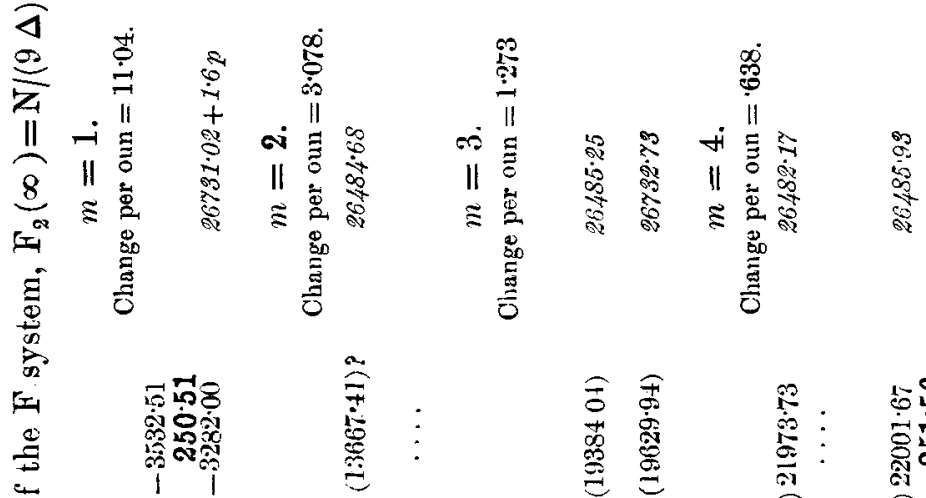

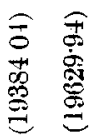

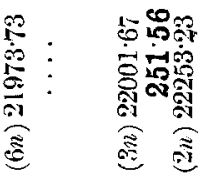

总
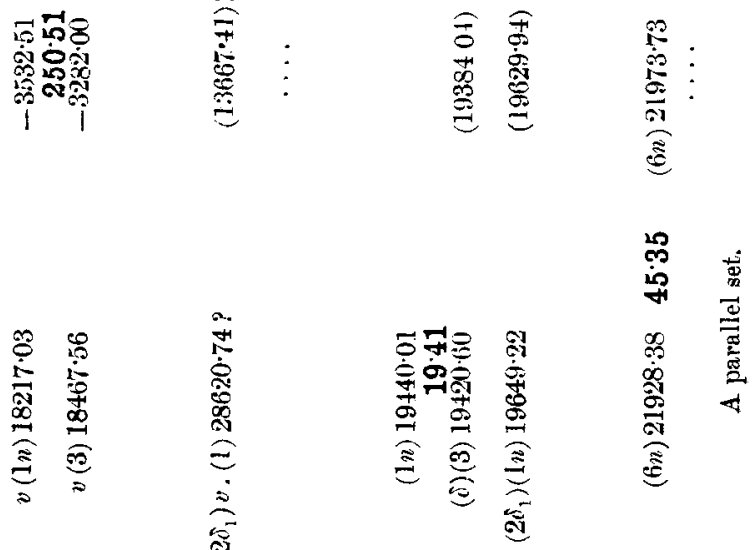

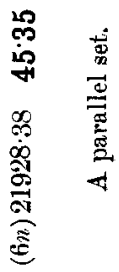

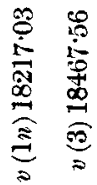

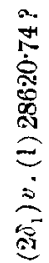




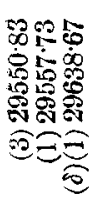

II

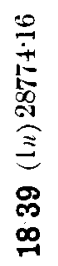

똥

فํํㅇ

है용

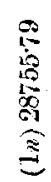

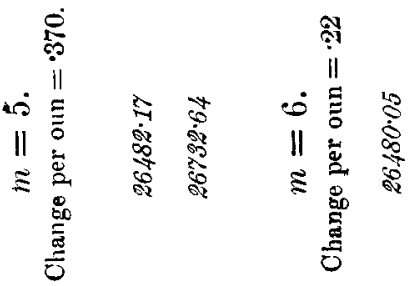
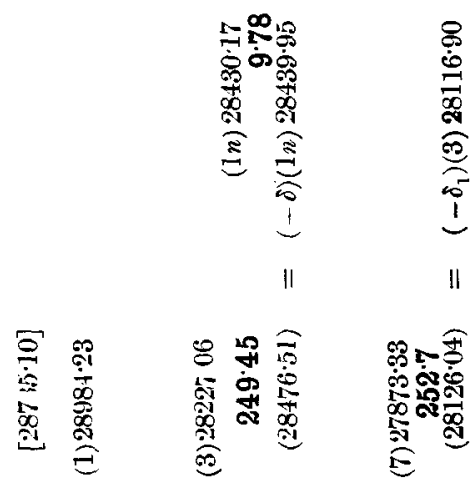

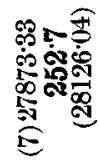

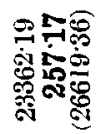
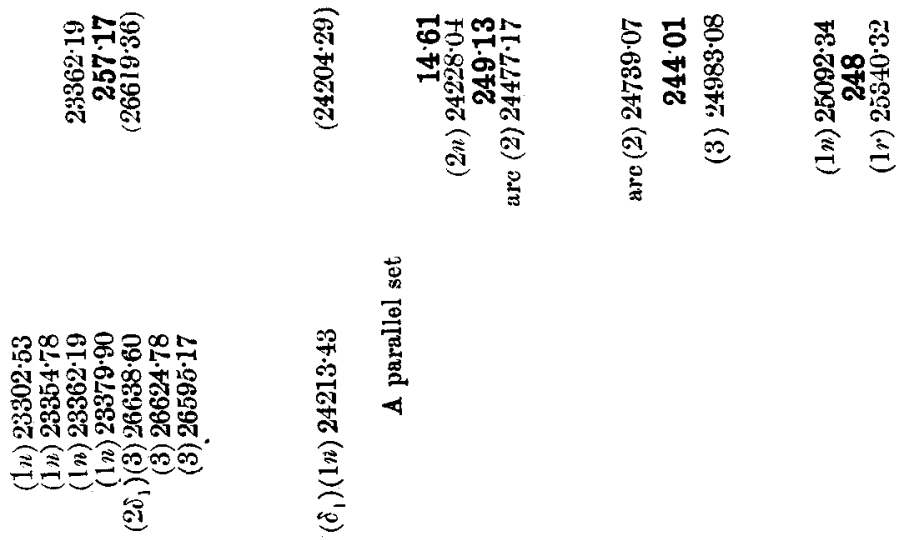
Notes. $-m=2$. The $F$ lines are in the unobserfed red region. There appear no simple $e, u, v$ links. That suggested is very problematical.

$m=3$. The separation $19 \cdot 41$ suggests $15 \delta_{1}$ on the sequent, which gives $19 \cdot 09.2 \delta_{1}$ on the limit gives $18 \cdot 24$ and is too far out. In connexion with $F_{1}$ there appear several lines which suggest displacement.

(5) $33522 \cdot 63$

\begin{tabular}{|c|c|c|c|}
\hline$(33586 \cdot 45)=F_{1}$ & $\begin{array}{r}72.96 \\
\text { (1) } 33595.59\end{array}$ & $\begin{array}{c}2 \delta \text { on limit gives } \\
\left(-\delta, \mathbf{F}_{1}\right.\end{array}$ & $73 \cdot 12$ \\
\hline $32 \cdot 88$ & $\begin{array}{r}(2 r) 33619 \cdot 33 \\
33 \cdot 60\end{array}$ & $\left(-3 \delta_{1}\right) \mathbf{F}_{1}\left(-5 \delta_{1}\right)$ gives & $33 \cdot 77$ \\
\hline 66.48 & $\begin{array}{r}(2 n) 33652.93 \\
76.62 \\
(4 n) 33729.55\end{array}$ & $\left(-6 \delta_{1}\right) \mathbf{F}_{1}\left(-10 \delta_{1}\right)$ gives & 67.54 for 66.48 \\
\hline
\end{tabular}

$m=4$. The only directly observed line is $F_{1}$. A precisely similar line lies next before it separated by 45.35 , which is $5 \delta_{1}(=45 \cdot 70)$ on the limit. They are clearly associated, but whether in this way, or with concomitant displacement in the sequence cannot be definitely settled with this order $m=4$ as the change per oun in the sequent is so small.

$m=5$. Here again there appear several sets of displaced lines. The different separations 257,242 , show that the sequents in the $\mathrm{F}_{2}$ set are different from those of $\mathrm{F}_{1}$-in other words, the sequent in $\mathrm{F}_{2}$ has experienced a change $\frac{1}{2}(257 \cdot 17-242 \cdot 78)=7 \cdot 20$. As $5 \delta=20 \delta_{\mathrm{k}}$ produces $7 \cdot 40$, it may be this. It makes the actual separation $249 \cdot 98$.

$m=6$. The separation of the associated $F_{1}$ lines, $18 \cdot 39$, is exactly due to a $2 \delta_{1}$ displacement on the limit.

The set given for $m=8$ is not in step for this order. The denominator of its sequent is 8.872840 , and is smaller than we should expect, although it is possibly in step with a rapid decrease foreshadowed in $m=7$, and analogons with $\mathrm{D}$ lines of highest order in the alkalies. It is given, bowever, as illustrating the general remarks on these series above. As an example of another set in this neighbourhood may be taken : 
It may be noticed that there are in the foregoing many instances where displacement on the sequent produces in nebulous line, whilst on the limit it is slarp. We cannot say, however, whether this is a general rule. 'To indicate that the given lines are at least near positions for normal lines, the following values of the denominators calculated from them are given: $1 \cdot 911560,2 \cdot 926185,3 \cdot 929447,4 \cdot 931517,5 \cdot 930130$, $6 \cdot 936977,7 \cdot 929360$.

\section{$\mathrm{P}$ and $\mathrm{S}$ Series.}

It has generally been assumed that the strong pair in the ultra violet, whose W.N. are (10) $411.72 .94,(10) 37357 \cdot 62$ with separation $3815 \cdot 32$, form the first $P$ doublet, in analogy with the strong violet pairs in $\mathrm{Cu}$ and $\mathrm{Ag}$. Although at tirst sight this assumption would appear natural, it is easy to show that it cannot be sustained. In the first place-as was pointed ont by Kayser and Runge in their original measures, -they are not in step with the $\mathrm{Cu} \mathrm{P}$ and $\mathrm{Ag} P$ lines. 'The $\mathrm{P}_{1}$ lines would run $\mathrm{Cu}, 30782 ; \mathrm{Ag}, 30471$ : Au, 41172 with Au much larger instead of somewhat smaller than Ag. But. the matter is definitely settled against the supposition when the question of the limit is considered. The rule that $\mathrm{S}(\infty)=p(1)$ is always so closely observed that it may be considered as absolute. The rule that $\mathrm{P}(\infty)=s(1)$, although never exactly fulfilled when $s(1)$ is extrapolated from tho $s(m)$ formula, is also so very nearly followed that it should be regarded as holding very approximately. In the case of gold $\mathrm{S}(\infty)=29469 \cdot 85=\mathrm{N} /(1.929145)^{2}$. If 41172 is $\mathrm{AuP}_{1}(1), \mathrm{P}(\infty)=7064279=\mathrm{N} /(1 \cdot 246015)^{2}$. The undoubted $S(2)$ lines (see below) give $s(2)=N /(2.545878)^{2}$. It is clear, therefore, that $s(1)$ cannot be $N /(1 \cdot 246)^{2}$ or near it, so that 41172 cannot be $P_{1}(1)$, or belong to a principal series of the ordinary type. Nevertheless, if regarded as belonging to a series in which the separations diminish with ascending order, it is possible to arrange a set for whose real existence as a definite series some evidence can be adduced, apart from the fact that the set are reproducible by a formula of the ordinary type. I give the evidence, such as it is, premising that considerations to follow may throw doubt on its validity. The letter $\mathrm{P}^{\prime}$ will be used provisionally to denote the series, as its type is undecided.

The value of $P(\infty)$ is $70642 \cdot 79+\xi$, and the denominator of the first sequent $=2 \cdot 940001-115 \cdot 9 \xi$. We should, therefore, expect to find $\mathrm{P}^{\prime}{ }_{1}(2)$ with a denominator near $3 \cdot 94 \ldots$, or wave number near 58000 . Also we might hope to find evidence in support by the combination line $p(1)-p(2)$. 
We find in this region several strong lines, the strongest being (5) $57954 \cdot 22(\lambda=1725 \cdot 5)$. 'This would make the combination line $p_{1}(1)-p_{1}(2)=16781 \cdot 28$, and we find it in the observed line (4) 16781.73 ( $\lambda=5957)$. We may, then, conclude with certainty that this allocation is correct. With these given lines for $m=1,2$, the formulæ constants are given by

$n=70642 \cdot 79+\xi-\mathrm{N} /\left\{m+\cdot 950924-199 \xi-\frac{021769-166 \xi}{m}\right\}^{2}$

This gives for $m=3 \ldots 8$, the following wave numbers $63590 \cdot 85,66148 \cdot 38,67542 \cdot 50,68370 \cdot 55,68906 \cdot 70,69273 \cdot 06$, all in the ultra violet beyond the observed region. For evidence as to the existence of these, it will therefore be necessary to have recourse to sounding, and to testing for the existence of combination lines.

$m=3$. The $e$ link requires $46337 \cdot 90$. There is obs. $(3 n) 46342 \cdot 43 \pm 2 . \quad d \lambda=-\cdot \cdot 1$

$p_{1}(1)-p_{1}(2)$ requires $22417 \cdot 21$ and is not observed.

$m=4$. The $e$ requires $48896^{\circ} 1$. There is obs.

The comb. requires $24975 \cdot 44$. There is

(5) $48892 \cdot 82 . \quad d \lambda=+\cdot 07$ obs. (3) $24977 \cdot 02$.

This combination gives $66149 \cdot 96$.

$m=5$. The $v$ requires $52608 \cdot 45$. There is obs.

(2) $52606 \cdot 66$.

The comb. requires 26369 , and is not

$$
d \lambda=-\cdot 03
$$

$$
d \lambda=\cdot 04
$$
observed.

$m=6$. The $e$ requires $51118 \cdot 2$. There is obs.

The comb. requires $27197 \cdot 6$. There are

(3) $51119 \cdot 52 . \quad d \lambda=-\cdot 12$ obs. (1) $27191 \cdot 28$, (1) $27202 \cdot 44$, of which the latter may be $D_{12}(6)$, but agrees well as a combination.

$m=7$. The $v$ requires 53972 65 . Obs. (4) 53978 $16 . d \lambda=-1$

The e requires 51654.4 . Obs. (1) $51659 \cdot 56 .=-1$

Both point to a common line $68911 \cdot 7$.

The comb. requires 27733.76 . There is

obs. (L) $27731 \cdot 94$.

$m=8$. The $v$ requires 54339 . Obs. (3) $54330 \cdot 11$.

The e requires $52020 \cdot 3$. Obs. (7) $52018 \cdot 31$, but the latter is too strong to be accepted as really related.

The comb. requires $28100 \cdot 0$. There is obs. $(2 r) 28104 \cdot 65$, which is also possibly $\mathrm{D}_{11}(8)$. 
Several strong lines appear in positions suitable for $\mathrm{P}_{2}(2)$, and they are probably related. The line ( 4 ) 56911 96 , regarded as a $\mathrm{P}_{2}^{\prime}$ line, has its mantissa less than that of $\mathrm{P}^{\prime}{ }_{1}(2)$ by $113800+389 p_{1}-333 p_{2}$. The lines are measured to the nearest unit in the first decimal point-consequently, if no observation errors are made, they are indefinite to the extent of $\cdot 05$, or in the above $p_{1}$ and $p_{2}$ are equally probable within \pm 5 The mantissæ difference is then with great probability exactly $\Delta, i$. e. the same as for $\mathrm{P}^{\prime}(1)$. We inight be tempted, therefore, to allocate it to the real $\mathrm{P}_{2}^{\prime}(2)$, were it not that the rule-so far as may be judged from the cise in the alkalies - appears to be that this difference is not the same for successive lines, but that it is about $\cdot 8$ to $9 \Delta$

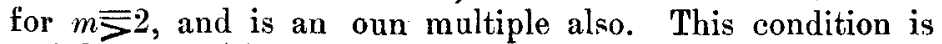
satisfied by (4) $56947 \cdot 61$ whose displacement from $P^{\prime}{ }_{1}(2)$ is 110106. As 78 $\frac{1}{2} \delta=\Delta-2 \frac{3}{4} \delta=110076$, this satisfies the multiple law with no observation error. None of the lines in this neighbourhood appear to give observed combination lines with $\mathrm{P}_{2}^{\prime}(1)$. The nearest is (2) 57191.88 requiring $19834 \cdot 26$ as against an observed ( $1 n) 19828^{\circ} 64$, possibly within error limits, especially as these combination lines seem susceptible to change by the electric field in the arc. It would seem that the $\mathrm{P}^{\prime}{ }_{2}$ lines here are represented by several associated lines, of which $56947 \cdot 61$ would appear to be the normal line.

In the following the estimated separations are based on the $78 \frac{1}{4} \delta$ displacement.

$m=3$. Separation about 394 or less. This is satisfied by a $v$ linked line and a combination, viz. :

(1) $48254 \cdot 39 v=63188 \cdot 44$.

$p_{2}(1)-p_{2}(3)=(1) 25834 \cdot 32$ gives $63191.94=\mathrm{P}_{2}^{\prime}(3)$.

$m=4$. Separation about 200 . Satistied by

(2n) $38695 \cdot 80 . e=65948 \cdot 11$.

$p_{2}(1)-p_{2}(4)=(2) 28595 \cdot 47$ gives $65953 \cdot 09=\mathrm{P}_{2}^{\prime}(4)$.

$m=5$. Separation about 114 .

(5) $52476 \cdot 4 \cdot v\left(-\delta_{1}\right)=67424 \cdot 23$.

(2) $50169 \cdot 9 . e \quad=67422 \cdot 2$.

$p_{2}(1)-p_{2}(5)=(1) 30067 \cdot 59$ gives $67425 \cdot 21=\mathrm{P}_{2}(5)$.

$m=6$. Separation about 70 .

(2) $51051 \cdot 66 . e=68303 \cdot 97$.

$p_{2}(1)-p_{2}(6)=(4 n) 30944^{\circ} 04$ gives $68301 \cdot 66=\mathrm{P}_{2}(6)$.

$m=7,8$. Separations about $48,33 \cdot 7$. 
The lines are collected in the following table, together with the deduced wave-lengths :-

\begin{tabular}{|c|c|c|c|}
\hline $1\left\{\begin{array}{l}10 R \\
10 R\end{array}\right.$ & $\begin{array}{c}\lambda . \\
2676 \cdot 05 \\
2428 \cdot 06\end{array}$ & $\begin{array}{c}n . \\
37357 \cdot 62 \\
41172 \cdot 94\end{array}$ & $p(\mathbf{1})-p(m)$ \\
\hline $2\left\{\begin{array}{l}4 \\
5\end{array}\right.$ & $\begin{array}{l}1756^{\circ} 0 \\
1725 \cdot 5\end{array}$ & $\begin{array}{l}56947 \cdot 61 \\
57954 \cdot 22\end{array}$ & (4) $16 \overline{781 \cdot 73}$ \\
\hline 3 & $\begin{array}{l}1582 \cdot 48 \\
1572 \cdot 5\end{array}$ & $\begin{array}{l}63191 \cdot 94 \\
63590 \cdot 80\end{array}$ & $\begin{array}{c}(1) 25834 \cdot 32 \\
\ldots \ldots\end{array}$ \\
\hline 4 & $\begin{array}{l}1516 \cdot 23 \\
1511 \cdot 16\end{array}$ & $\begin{array}{l}65953 \cdot 07 \\
66149 \cdot 96\end{array}$ & $\begin{array}{l}\text { (2) } 28595 \cdot 47 \\
\text { (3) } 24977 \cdot 02\end{array}$ \\
\hline 5 & $\begin{array}{l}1483 \cdot 19 \\
1480 \cdot 5\end{array}$ & $\begin{array}{l}674.25 \cdot 21 \\
67540 \cdot 65\end{array}$ & $\begin{array}{c}\text { (1) } 30067.59 \\
\ldots\end{array}$ \\
\hline 6 & $\begin{array}{l}1464 \cdot 09 \\
1462 \cdot 75\end{array}$ & $\begin{array}{l}68301 \cdot 68 \\
68369 \cdot 80\end{array}$ & $\begin{array}{c}(4 n) 30944 \cdot 04 \\
\text { (1) } 27202 \cdot 44 \text { ? }\end{array}$ \\
\hline 7 & $\begin{array}{l}\ldots \ldots \\
1451: 28\end{array}$ & 6390488 & (2) 27781.94 \\
\hline 8 & $\ddot{144396}$ & $\ddot{69979 \cdot 38}$ & $(2 r) 2810465 ?$ \\
\hline
\end{tabular}

The considerable number of combination lines of a very common type $p(1)-p(m)$ strongly supports the supposition that the set form an actually related series. The question presents itself whether the objection raised as to the limit not being $s(1)$ can be met. In the foregoing treatment of the set as a $\mathrm{P}$ series, 29469 is $p(1)$, and the series is regarded as produced by deducting the sequence $p(m)$ from a limit $7064 \%$. We have shown that 70642 cannot be the true $\mathrm{P}(\infty)$ or $s(1)$. This difficulty may, however, be possibly explained by regarding 70642 as composite-in other words, by regarding the series in question as linked to the true $\mathrm{P}$ series and $70642=$ $P(\infty)+$ links (that is, as forming a parallel series to the $P$ ). For instance, il it was the single $e$ link the $\mathrm{P}(\infty)$ would be $70642 \cdot 79-17252 \cdot 31=53390 \cdot 68=\mathrm{N} /(1 \cdot 4335)^{2}$, which is within possible reach of $s(1)$. In this connexion the large number of $-v$ links noted above in sounding is suggestive. To these may be added the following for the orders $m=1,2$, in which also the linkages $v+c$ are included :-

(2r)21629.62 4606.98 (2n) $26236 \cdot 60$ 14936.34 $41172 \cdot 94 \quad \mathrm{P}^{\prime}{ }_{1}(1)$

(1) 37410.034606 .60 (1) $42016.6314930 .98+3.3 \rho 56947 \cdot 61+3 \cdot 3 p \mathrm{P}_{2}^{\prime}(2)$ $(1 n) 38418 \cdot 544606+14929 \cdot 68+3 \cdot 3 p 5795+22+3 \cdot 3 p \mathrm{P}_{1}^{\prime}(2)$ 
in which $p$ is equally probable within $\pm \cdot 5$ and possible within \pm 2 . The linkage $v+c=14934 \cdot 05+4607 \cdot 77=$ 19541.82 here indicated would give for the $\mathrm{P}(\infty)=s(1)$ a value $70642 \cdot 79-19541 \cdot 82 \cdot 51101=\mathrm{N} /(1 \cdot 464 \ldots)^{2}$. It is, in fact, a value obtained by an independent attack on the $P$ problem undertaken below. It may be noted that by an explanation of this kind the combination lines given by the $\mathrm{P}^{\prime}$ set will be the same as by a $\mathrm{P}$ set, and consequently $p(1)-p(m)$.

There is also another way in which the presence of the $S(\infty)$ in the 41172 pair may be accounted for, and in our present state of ignorance as to spectral constitution, it is advisable to consider it. It is to regard the 29469 as a limit and the pair as summation lines. Now it is curious that such a set can be found. They are

$$
\left.\begin{array}{lr}
(3) 21558 \cdot 05 & (4 \delta)=.81 \cdot 86 \\
(3) 21577 \cdot 30 & \left(3 \delta_{1}\right)=. .81 \cdot 76 \\
(3) 21589 \cdot 65\left(-5 \delta_{2}\right) & =.82 \cdot 21
\end{array}\right\}(21581 \cdot 94) \quad 29469 \cdot 78 \quad(10) 37357 \cdot 62
$$

3814.83

\section{$3815 \cdot 32$}

\section{(1n) $25396 \cdot 77 \quad 33284 \cdot 85 \quad(10) 41172 \cdot 94$}

Denoting the series by $R$ for convenience, the $R_{1}$ is given as split up into at least three displaced lives. The sequent $(37357-29469)$ has denominator $3 \cdot 728865$, so that the lines correspond to $R(3)$. The oun displacement on the sequent produces $1 \cdot 488$. The three lines displaced from $R_{1}(3)$ as indicated give the same value for $\mathrm{R}(3)$ within fractions of possible errors, and are of the same intensity and character. Indeed, they would seem to afford a striking illustration of the split up of a normal line of strong intensity (witness, the intensities of the $\mathbf{R}$ lines) into a number of weaker displaced ones *.

For $m=4$ the denominator should be near $4 \cdot 72$, and the following set satisfying the conditions are found:
(1) $24558 \cdot 6 \mathrm{l}$
$29467 \cdot 30$
$(34376 \cdot 11)=\left(-6 \delta_{1}\right)(10) 34311 \cdot 55 ?$
$3814 \cdot 93$
$3815 \cdot 88$
(3) 28373.54
จீ3刃827\%
(2) $38191 \cdot 99$

* There is, in fact, a whole successive group of similar character-in addition to the above may be adduced (3) $21530: 70\left(34 \delta_{1}\right)=\ldots 81 \cdot 30$; (1) $21542 \cdot 49\left(26 \delta_{1}\right)=\ldots 81 \cdot 18 ;(3) 21601 \cdot 77\left(-13 \delta_{1}\right)=\ldots 82 \cdot 43$, a mean from the six of $21581 \cdot 79$ making $\nu=38150$. 
The mean limits are both in defect by about $2 \cdot \bar{y}$, and the allocation of $\boldsymbol{R}_{1}$ seems rather arbitrary. $\mathbf{R}_{2}$ is much weaker than $\mathbf{R}_{1}$ and can be accounted for by the presence of the displaced lines, thus :

(2) $38127 \cdot 63$

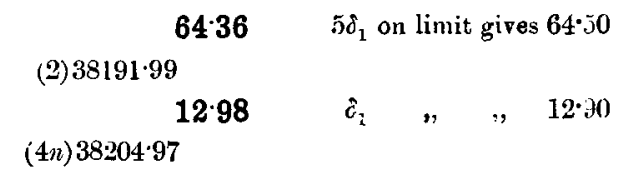

From the sets for $m=3,4$ and the given $\mathrm{S}(\infty)$, the following formula is found :

$$
n=29469 \cdot 85-\mathrm{N} /\left\{m+\cdot 715861+\frac{\cdot 039012}{m}\right\}^{2} .
$$

The positive term in $1 / \mathrm{m}$ points to the series being of the $\mathrm{D}$ or $\mathrm{F}$ type. With this the set for $m=5$ is found to be $(\mathrm{O}-\mathrm{C}=-\cdot 3)$.

$$
\begin{aligned}
& \left.\begin{array}{ll}
(2) 26112 \cdot 25(-4 \delta) & =.19 \cdot 84 \\
(8) 26130 \cdot 61 & =. .19 \cdot 85
\end{array}\right\} 26119 \cdot 8 t \quad 29471 \cdot 12 \quad \text { (2) } 32822 \cdot 40 \\
& 3516 \cdot 13 \\
& \text { (1) } 36338 \cdot 53
\end{aligned}
$$

For $m=2, \mathrm{R}_{1}$ comes into the ultra red, but the others are indicated, viz. :

$\begin{array}{rcc}{[14815 \cdot 91]} & 29469 \cdot 60 & (44123 \cdot 29)=(1 n) 44130 \cdot 82\left(2 \hat{o}_{1}\right) \\ 3815 \cdot 56 & & 3814 \cdot 20 \\ (2 n) 18638 \cdot 98\left(-2 \delta_{1}\right)=(18631 \cdot 47) & 33284 \cdot 48 & (\mathbf{1}) 47937 \cdot 49\end{array}$

Any combination lines from these would fall in the ultra red region. There are thus considerable indications in favour of this second explanation, although perhaps not very convincing. Both cannot be correct and one, at least, is therefore spurious. I have inserted the second because it affords warning against relying on a fow numerical coincidences, especially where, as in this case, so many of the lines are in crowded positions. At the same time, the second may be possibly the real solution, and the question may be left for solution in the future.

We may make the direct attempt to determine the $P$ series without regard to the 41172 , which, as we have seen, cannot 
belong to a normal $\mathrm{P}$. We require a doublet with separation 3815 , such that the sum of the larger W.N. and 29469 shall show a mantissa within possible reach of $s(1)$. There are only two observed in the region involved, viz. :
(2n) $17879 \cdot 43$
(3) 17573.91
3816.92
3818.66
(6) $21696 \cdot 35$
(6) $21392 \cdot 57$

both spark lines not seen in the arc. They are possibly, therefore, both $\mathrm{P}$ doublets modified in some way, as is indicated also by the enlarged separations. Since $21696.35+$ $29469 \cdot 85=51166 \cdot 20=\mathrm{N} /\{1 \cdot 464071\}^{*}$, the $\mathrm{P}(\infty)$ is near a possible $s(1)$. Both the limit 51166 and the line 21696 are near the values given by the $v+c$ linkage in the $\mathrm{P}^{\prime}$ series. The lines given in the following table seem to give at least one set of $\mathrm{P}$ series. The pair for $m=2$ are both seen in the arc, but are enhanced in the spark. The formula for $P_{1}$ found from the first and second is

$$
m=51166 \cdot 20-\mathrm{N} /\left\{m+\cdot 978446-\frac{\cdot 049298}{m}\right\}^{2} .
$$

This gives for $m=3,44179 \cdot 44$ with $\mathrm{O}-\mathrm{C}=-\cdot 1$. For $m=4$, $n=46719 \cdot 15$ the nearest observed line being ( $1 n) 46703 \cdot 65$.

For the first two orders any $\mathbf{P}$ lines would lie in the unobserved ultra violet. For $m=3$ there are seen two which serve for $\mathbf{P}(3)$ lines, in which it may be noticed that the means give a larger limit than that assumed, but one in accordance with the enlarged separations observed. A displacement $\delta_{1}$ in $S(\infty)$ increases $\nu$ by $2 \cdot 17$ to 3817.73 and $S(\infty)$ by $10^{\circ} 76$. Hence $P(\infty)$ should be 51176.96 , which duly appears for $\mathrm{P}_{2}(3), \mathrm{P}_{2}(3):-$

P.

$$
\begin{aligned}
& m=I\left\{\begin{array}{r}
(2 n) 17870 \cdot 43 \\
(6) 21696 \cdot 35
\end{array}\right. \\
& n=2\left\{\begin{array}{r}
(8) 37844 \cdot 09 \\
(6) 38595 \cdot 88
\end{array}\right. \\
& n=3\left\{\begin{array}{l}
(4 n) 43892 \cdot 19 \\
(4 n) 44181 \cdot 90
\end{array}\right.
\end{aligned}
$$

P.

Outside observed region.

$$
\begin{aligned}
& 51175 \cdot 60+1 \cdot \% p \\
& 51169 \cdot 17+1 \cdot y_{p}
\end{aligned}
$$$$
\text { (3) } 58459 \cdot 02
$$$$
\text { (4) } 58156 \cdot 44+3 \cdot 3 p(-200,5 \tau)
$$

The S Srries. The $\mathrm{S}(2)$ set are quite definitely $13309 \cdot 98$ (corrected from Lehmann's $13312 \cdot 9+13$ ) and $17125 \cdot 54$, both from their analogous position with the $\mathrm{S}(2)$ lines in $\mathrm{Cu}, \mathrm{Ag}$ 
and because the Zeeman pattern for 17125 is that of a $S_{2}$ line. It gives with the known $S(\infty)$ a denominator 2.545 . We can now proceed in one of two ways, (1) either by using $5116 t^{\circ}=\mathrm{P}(\infty)$ as $s(1)$ or $(2)$ by looking for a 3815 doublet in the region given by a denominator 3.54. Method (1), however, never gives a formula reprod ucing well other lines. Here it prophesies a line $S_{1}(3)$ at 20879 . There is only one line in this neighbourhood, viz. (6) 20858 already adopted as $\mathrm{D}_{22}(2)$ and showing no companion at 3815 ahead. There is, however, in the 3.54 neighbourhood one doublet which satisfies the condition, although their Zeeman patterns, so far as they can be deduced from Hartmann's measures, are indeterminate. They are (3) 20776503815.57 (6) 24592.07. The resulting formula for $S_{1}$ is

$$
n=29469 \cdot 85-\mathrm{N} /\left\{m+\cdot 563932-\frac{\cdot 036108}{m}\right\}^{2} .
$$

The lines as indicated through this formula are given in the following table :-

S.

S.

$$
m=2 \text {. }
$$

Change per oun $=4 \cdot 808$.

$\begin{array}{lll}\text { (4) } 13309 \cdot 98 \quad 29471 \cdot 77 & \text { (1) } 45633 \cdot 56+2 \cdot 08 p\end{array}$

$3815 \cdot 56$

(6) $17125 \cdot 54$

$$
m=3 \text {. }
$$

Change per oun $=1 \cdot 723$.

$\begin{array}{ccc}(5) 20776 \cdot 50 & 29469 \cdot 44 & (38162 \cdot 38) \\ \mathbf{3 8 1 5} 57 & \mathbf{3 8 1 5} \cdot 45\end{array}\left\{\begin{array}{l}(2) 38127 \cdot 63(-50)=62 \cdot 09 \\ (5) 38191 \cdot 99\left(17 \delta_{1}\right)=62 \cdot 70\end{array}\right.$

(10)24592.07 $38284 \cdot 95 \quad(41977 \cdot 83)\left\{\begin{array}{l}(4 n) 41.960 \cdot 21\left(-10 \delta_{1}\right)=77 \cdot 40 \\ (1 n) 41995 \cdot 45 \quad\left(10 \delta_{1}\right)=78 \cdot 21\end{array}\right.$

$$
m=4 \text {. }
$$

Change per oun $=814$.

\section{$\left(\delta_{1}\right)(1) 27987 \cdot 13=27974 \cdot 23 \quad 33285 \cdot 13 \quad(5) 38596 \cdot 04$}

These wave numbers are $34 \cdot 61$ less than the calculated $S_{2}$, but they give a very exact $\frac{1}{2}(\mathrm{~S}+\mathbf{S})$ and therefore indicate displaced $s(4)$. A displacement of $42 \delta_{1}$ on sequent gives $34 \cdot 19$. $\mathrm{O}-\mathrm{C}=-\cdot 05$. 


$$
m=5 \text {. }
$$

Change per oun $=448$.

(2r) $2592: 38$

$\mathrm{O}-\mathrm{C}=-66 \quad \ldots \ldots$

3818.90

(1) $29741 \cdot 28$

$$
\begin{array}{rr} 
& \multicolumn{2}{c}{m=6 .} \\
\left(-\delta_{1}\right)(1) 26 \% 10 \cdot 66 & =(26921 \cdot 42) \quad 0-\mathrm{C}=-\cdot 2 t \\
3813 \cdot 38 & 3815 \cdot 52 \\
\left(-\delta_{1}\right)(2 r) 30724.04 & =(30736.94)
\end{array}
$$

This case is specially interesting as the observed lines treated as $\left(\delta_{1}\right) \mathrm{S}$ give also the separation proper to the limit $\left(\delta_{1}\right) \mathbf{S}(\infty)$.

Amongst the results obtained in the foregoing discussion of most importance to the general theory of spectral analysis may be mentioned :-

(1) The existence of a $\mathrm{D}$ set corresponding to order $\boldsymbol{m}=1$ far up in the ultra red whose satellite mantissa conforms to the general rule of being a multiple of $\Delta$. Gold therefor" does not form an exception, as formerly appeared to be the case.

(2) The indication given of the existence of summation lines for $P$ and $S$ series. The evidence for these lines in [V.] is drawn from $D$ and $F$ series. In general, such lines for $\mathbf{S}$ and $P$ will lie far down in the ultra violet, and this is, no doubt, one reason why they lave not previously been recognized. The evidence here given must be sustained by further numerical coincidences obtained in other spectra before it is regarded as conclusive, but it is sufficient to render it extremely probable.

(3) The more accurate determination of the oun depending on $81 \delta=\Delta=113951-6 p$, where $\cdot 1 p$ angstroms is an observational error. This result gives

$$
\delta=1406 \cdot 802-\cdot 074 p \text {. }
$$

Taking the ratio of $\delta$ to $(\mathrm{W} / 100)^{2}$, where $\mathrm{W}$ is the atomic weight, to be $361 \cdot 75+\cdot 05 q$, the resulting value of the atomic weight of gold is

$$
197 \cdot 2024-\cdot 0052 p-\cdot 0136 q .
$$

The University,

April 12, 1919.

$$
\text { Sheffield, }
$$

